S. 44. J. B. Lippincott, Philadelphia (1966). - 7. RrNGorR, S., Thesis, Univ. of Gent, 1967. Toepassing van de Gedifferentieerde LDH Bepaling bij experimentele en klinische Nieraandoeningen. Arscia Uitgaven N. V., Brussel (1967). - 8. RrNGorR, S., in: Aktuelle Probleme der klinischen Biochemie, edited by H. Aebi, H. Mattenheimer, F. W. Schmidt. Vol. II. International Conference on enzymes in the urine. Hans Huber, Bern und Stuttgart, (1968). - 9. Wiene, R. J. und Y. van MaERcke, Ann. N. Y. Acad. Sci. 94, 898 (1961). - 10. Bonting, S. L., V. E. Pollak, R. C. MuehrCKe und R. M. Kark, Science Washington 127, 1342 (1958). - 11. Pollak, V. E. und H. Matrenheimer, Arch. Int. Med., Chicago 109, 473 (1962). - 12. MatTenmheier, H., V. E. Pollak, R. C. Muehrcke und R. M. Kark, Verh. Dtsch. Ges. inn. Med. 70, 579 (1964). - 13. Matrenhermer, H., in: Aktuelle Probleme der Klinischen Biochemie, edited by H. Aebi, H. Mattenheimer, F. W. Schmidt. Vol. I. Praktische Enzymologie. Hans Huber, Bern u. Stuttgart (1968). - 14. ThIELE, K. G. und H. MATTENHEIMER, this journal 4, 232 (1966). - 15. KENNY, M. A., $\mathrm{Ph}$. D. Thesis. The Graduate College, Univ. of Illinois at the Medical Center Chicago, 1967. Relationship of an electrophoretically separable variant to lactic dehydrogenase isoenzyme $2\left(\mathrm{H}_{3} \mathrm{M}\right)$ from human erythrocytes. - 16. KENNY, M. A. und M. E. RAFELSON, Presbyterian-St. Luke's Hospital Med. Bull. 6, 92 (1967). - 17. MatTenheimer, H., Mikromethoden für das klinisch-chemische und biochemische Laboratorium. 2. Aufl., Walter de Gruyter \& Co., Berlin (1966). - 18. Starkweather, W. H., L. Cousinkau, H. K. Schoch und C. J. Zarafonetis, Blood 26, 63 (1965). - 19. Cahn, R. D., N. O. Kaplan, L. Levins und E. Zwilling,
Science Washington 136, 962 (1962). - 20. DAwson, D. M., T. L. GoodfrIEND und N. O. KAPLAN, Science Washington 143, 929 (1964). - 21. KEMP, E., in: Aktuelle Probleme der klinischen Biochemie, edited by H. Aebi ,H. Mattenheimer, F. W. Schmidt. Vol. II. International Conference on enzymes in the urine. Hans Huber, Bern u. Stuttgart (1968). - 22. PlagemanN, P. G. W., K. F. Gregory und F. WroblewsKr, J. biol. Chemistry 235, 2288 (1960). - 23. Veseld, E. S., Science Washington 150, 1590 (1965). - 24. LINDY, S. und M. Rajasalm, Science Washington 153, 1401 (1966). - 25. WALLER, H. D., in: Erbliche Stoffwechselkrankheiten, edited by F. Linneweh, p. 74. Urban und Schwarzenberg, München-Berlin (1962). - 26. LEE, J. B., V. K. VANCE und G. F. Cahilt, Amer. J. Physiol. 203, 27 (1962). - 27. György, P., W. KELLER und T. BreHME, Biochem. Z. 200, 356 (1928). - 28. Dickens, F. und H. WeIL-Malmerbe, Biochem. J. 30, 659 (1936). - 29. Davis, R. P., in: Progress in Pyelonephritis, edited by E. H. Kass. S. 408. F. A. Davis Company, Philadelphia (1965). 30. Davies, R. E., R. W. Winters und E. L. Kean, Federat. Proc. 21, 427 (1962). - 31. Kramer, K., K. Thurau und P. Deetjen, Pflügers Arch. ges. Physiol. 270, 251 (1960). - 32. Ulirich, K. J., Erg. Physiol. 50, 433 (1959). - 33. Mattenhermer, H., in: Aktuelle Probleme der klinischen Biochemie, adited by $\mathrm{H}$. Aebi, $\mathrm{H}$. Mattenheimer, F. W. Schmidt. Vol. II. International Conference on enzymes in the urine. Hans Huber, Bern u. Stuttgart (1968). 34. Jahnecke, J., G. Löffler, M. Meisch und E. Streichen, Klin. Wschr. 45, 466 (1967). - 35. Schreiner, G. E. und J. F. MAHER, Uremia: Biochemistry, Pathogenesis, Treatment. Charles C. Thomas, Springfield, Illinois, Section III.

Prof. Dr. H. Mattenheimer Presbyterian St. Luke's Hospital 1753 W. Congress Parkway Chicago, Ill., USA 60612

\title{
Kombinierte enzymatische Methode zur Bestimmung von 3-Hydroxykynurenin und 3-Hydroxyanthranilsäure in freier und veresterter Form im Harn
}

\author{
Von H. SCHIEVELbEIN und KARIN LöSCHENKoHL
}

Aus dem Klinisch-Chemischen Institut an der Chirurgischen Universitätsklinik München (Direktor: Prof. Dr. Dr. E. Werle)

(Eingegangen am 1. Februar 1968)

3-Hydroxykynurenin wird mit Kynureninase (L-Kynurenin-Hydrolase, EC 3.7.1.3) zu 3-Hydroxyanthranilsäure umgesetzt. In einer nachfolgenden Reaktion wird diese Verbindung mit 3-Hydroxyanthranilsäureoxydase (3-Hydroxyanthranilat : $\mathrm{O}_{2}$ Oxydoreduktase, EC 1.13.1.6) zu 2-Akrolein-3-aminofumarat oxydiert. Dieses Reaktionsprodukt kann bei $360 \mathrm{~nm}$ quantitativ bestimmt und so die Konzentration der beiden Tryptophanstoffwechselprodukte im Harn errechnet werden.

3-Hydroxykynurenine is degraded by kynureninase (L-kynurenine hydrolase, EC 3.7.1.3) to 3-hydroxyanthranilic acid. In a subsequent reaction, this compound is oxidised by 3-hydroxyanthranilic acid oxidase (3-hydroxyanthranilate : $\mathrm{O}_{2}$ oxidoreductase, EC 1.13.1.6) to 2-acroleine-3-aminofumarate. This reaction product can be estimated quantitatively at $360 \mathrm{~nm}$ and thus the concentration of the two tryptophane metabolites can be calculated.

Seit dem chromatographischen Nachweis von 3Hydroxyanthranilsäure ${ }^{1}$ ) im Harn von tuberkulösen Patienten nach oraler Tryptophanbelastung durch Musajo und Mitarbeiter (1) 1952 sind zahlreiche Veröffentlichungen erschienen, in welchen das Ausscheidungsmuster von Tryptophanmetaboliten bei verschiedenen pathologischen Zuständen beschrieben wird (Literaturübersicht bis 1964 bei Musajo und BeNASSI (2)). Eine besondere Bedeutuing erlangte die Bestim-

1) Abkürzungen: 3-OH-A = 3-Hydroxyanthranilsäure. 3-OH-K= 3-Hydroxykynurenin, PLP = Pyridoxal-5'-phosphat. mung von Tryptophanmetaboliten im Harn, nachdem Boyland (3) gefunden hatte, daß Patienten mit einem Carcinom der Harnblase gegenüber Normalen eine erhöhte Ausscheidung der im Tierexperiment carcinogenen Stoffwechselprodukte 3-OH-A und 3-Hydroxykynurenin aufweisen. Für die Bestimmung der erwähnten Substanzen im Harn wurden eine Reihe von Methoden angegeben, welche infolge ihres Mangels an Spezifität oder ihrès großen Aufwandes für die Untersuchung einer größeren $\mathrm{Zahl}$ von Proben nur bedingt geeignet sind. 
Für die Frage, ob 3-OH-K oder 3-OH-A eine kausale Bedeutung für die Ätiologie von Harnblasentumoren zukommt, ist es notwendig, die freien Verbindungen neben den mit Schwefel- oder Glucuronsäure veresterten Formen getrennt zu erfassen, da nur den unkonjugierten Verbindungen eine onkogene Aktivität zukommen dürfte. Die bisher vorliegenden Untersuchungen $z u$ diesem Problem ließen eine Beantwortung dieser Frage, offensichtlich wegen methodischer Schwierigkeiten, vermissen. Wir veröffentlichten vor kurzem eine Methode zur enzymatischen Bestimmung der 3-OH-A (4). Die vorliegende Arbeit beschreibt eine Methode, welche diese Bestimmung als Indikatorreaktion benutzt. Sie eignet sich zur Bestimmung der freien und, nach Hydrolyse, der Gesamtkonzentration von $3-\mathrm{OH}-\mathrm{K}$ und $3-\mathrm{OH}-\mathrm{A}$ im Harn.

\section{Prinzip}

3-OH-K wird durch das Enzym Kynureninase (LKynurenin-Hydrolase, EC 3.7.1.3) in 3-Hydroxyanthranilsäure und Alanin nach folgendem Reaktionsschema gespalten:<smiles>Nc1c(O)cccc1C(=O)CC(N)C(=O)O</smiles><smiles>CC(N)C(=O)O</smiles>

Die bei der Reaktion entstehende 3-OH-A wird mit 3-Hydroxyanthranilsäureoxydase (3-Hydroxyanthranilat: $\mathrm{O}_{2}$ Oxydoreduktase, EC 1.13.1.6) in einer nachgeschalteten Reaktion nach folgendem Reaktionsschema zu 2-Akrolein-3-aminofumarat umgesetzt:<smiles>NC(=O)C(/C=C\C(=O)O)=C(\N)C(=O)O</smiles>

Das Reaktionsprodukt, dessen Konfiguration von BONNER und YANOFSKY (5) vorgeschlagen und von Kuss (6) bestätigt wurde, besitzt ein Absorptionsmaximum bei $360 \mathrm{~nm}$ und kann bei dieser Wellenlänge quantitativ bestimmt werden. Der Umsatz von 3-OH-K wurde in Anlehnung an die Bestimmung der Aktivität der Kynureninase, wie sie von Weber und Wrss (7) beschrieben wurde, durchgeführt. Das Prinzip der Methode zur Bestimmung der 3-OH-A wurde bereits von Wiss (8) zur Bestimmung der Aktivität der 3Hydroxyanthranilsäureoxydase beschrieben.

\section{Methodik}

Reagentien

1. Phosphatpuffer, $0,033 \mathrm{M}, \mathrm{pH} 7,4$

2. a) 3-OH-K-Stammlösung:

$1,5 \mathrm{mg}$ 3-OH-K (Calbiochem, Los Angeles) werden in $50 \mathrm{~m} / 0,1 \mathrm{~N}$ $\mathrm{HCl}$ gelöst. Diese Lösung ist einige Wochen im Kühlschrank bei $4^{\circ}$ haltbar.

b) 3-OH-K-Gebrauchslösung:

$5 \mathrm{~m} /$ Stammlösung werden mit $5 \mathrm{~m} / 0,1 \mathrm{~N} \mathrm{NaOH}$ neutralisiert und mit Puffer (Reagenz 1) auf $50 \mathrm{ml}$ verdünnt $(3 \mu \mathrm{g} 3-\mathrm{OH}-\mathrm{K} / \mathrm{m} /$ ). Diese Lösung muß immer frisch hergestellt werden.

3. Pyridoxal-5'-phosphat-Lösung:

$6 \mathrm{mg}$ Pyridoxal-5'-phosphat werden in $100 \mathrm{~m} /$ Puffer (Reagenz 1) gelöst, diese Lösung ist etwa 1 Woche bei $4^{\circ}$ haltbar.

4. Enzyme:

a) Kynureninase aus Schweineleber wurde nach einer von Wrss und WeBER (9) angegebenen Methode einschließlich des Schrittes der fraktionierten Ammonsulfatfällung gereinigt. Unsere lyophilisierten Präparate wiesen, mit Kynurenin als Substrat, eine Aktivität von etwa $0,5 \mu \mathrm{g}$ gebildete Anthranilsäure/mg Protein/Std. auf. Je nach Aktivität des Enzympräparates werden 5-15 mg Enzym für einen Ansatz benötigt, welches in Substanz oder in Lösung den Ansätzen zugegeben werden kann.

b) 3-Hydroxyanthranilsäureoxydase aus Schweineleber wurde nach WIss und Mitarbeiter (10) gereinigt. Unsere Präparate wiesen eine spezifische Aktivität von 3-10 $\mu \mathrm{g}$ 3-OH-A umgesetzt/mg Protein/Min. auf.

Zum Ansatz werden 5-20 mg des lyophilisierten Präparates benötigt und in $1 \mathrm{~m} /$ Puffer (Reagenz 1) gelöst. Die gereinigten Enzympräparate sind sehr instabil und verlieren in Lösung schnell ihre Aktivität, die Lösung muß am Tage des Gebrauchs hergestellt und im Eisbad aufbewahrt werden.

c) $\beta$-Glucuronidase/Arylsulfatase:stabilisierte Enzymlösung (Boehringer $u$. Söhne, Mannheim).

5. Tris-Puffer $0,033 \mathrm{M}, \mathrm{pH} 7,4$.

6. Ferroammoniumsulfatlösung:

$210 \mathrm{mg} \mathrm{FeSO}{ }_{4} \cdot\left(\mathrm{NH}_{4}\right)_{2} \mathrm{SO}_{4} \cdot 6 \mathrm{H}_{2} \mathrm{O}$ (Mohrsches Salz) werden in $100 \mathrm{ml}$ Tris-Puffer (Reagenz 5) gelöst.

7. Cysteinlösung:

$240 \mathrm{mg}$ Cysteinhydrochlorid werden in $100 \mathrm{ml}$ Tris-Puffer (Reagenz 5) gelöst.

8. Cystein-Eisenlösung:

Je $1 \mathrm{~m} /$ Reagenz 6 und 7 werden miteinander vermischt. Die beiden Substanzen sind Aktivatoren der 3-Hydroxyanthranilsäureoxydase. Die Lösung muß tägl. frisch angesetzt wetden.

9. $\mathrm{NaOH}, 1 \mathrm{~N}$ und $0,1 \mathrm{~N}$.

10. $\mathrm{HCl}, 0,1 \mathrm{~N}$.

Durchführung

Vorbereitung der Harne:

Die beiden o-Hydroxyamine 3-OH-K und 3-OH-A sind bei neutralen und alkalischen $\mathrm{pH}-$ Werten sehr instabil (11), daher sollen Harnproben möglichst unmittelbar nach dem Sammeln verarbeitet werden. Wenn dies nicht möglich ist, lassen wir 24-Stdn.-Harnproben mit $\mathrm{HCl}$ auf ein $\mathrm{pH}$ von mindestens 4 bringen. Müssen Harnproben längere Zeit konserviert werden, so empfiehlt sich das Aufbewahren in eingefrorenem Zustand.

Für die Bestimmung der Gesamtkonzentration der Verbindungen im Harn wird dieser wie folgt hydrolysiert: $10 \mathrm{~m} l$ Harn werden mit $0,04 \mathrm{ml}$ der $\beta$-Glucuronidase/Arylsulfataselösung bei Raumtemperatur 2 Stdn. lang inkubiert. Eine längere Inkubationszeit bewirkt eine Abnahme der Konzentration der freien Verbindungen. Dann werden je $10 \mathrm{ml}$ des hydrolysierten und des nichthydrolysierten Harnes mit $1 \mathrm{~N} \mathrm{NaOH}$ auf ein $\mathrm{pH}$ von 7,4 eingestellt und filtriert. Die Bestimmung der 3-OH-A muß unmittelbar nach Einstellung des Harnes auf diesen $\mathrm{pH}$ durchgeführt werden, da die Verbindung bei diesem $\mathrm{pH}$ instabil ist. 


\section{Ansätze}

a) Zur Bestimumung von 3-OH-A

Die Ansätze werden, wie in Tabelle 1 beschrieben, in $3 \mathrm{~m} l$ Küvetten mit $1 \mathrm{~cm}$ Schichtdicke durchgeführt.

Tab. 1

Ansätze zur Bestimmung der 3-OH-A im Harn

\begin{tabular}{lcc}
\hline & $\begin{array}{c}\text { Analyse } \\
\text { (A) }\end{array}$ & $\begin{array}{c}\text { Standard } \\
(\mathrm{S}+\mathrm{A})\end{array}$ \\
\hline Tris-Puffer $(\mathrm{ml})$ & 1,00 & 1,00 \\
Harn (ml) & 1,00 & 1,00 \\
Standard (ml) & - & 0,05 \\
$0,1 \mathrm{~N}$ NaOH $(\mathrm{ml})$ & 0,02 & 0,05 \\
Cystein-Fe-Lösung $(\mathrm{ml})$ & 0,20 & 0,02 \\
Enzymlösung (ml) & 0,10 & 0,20 \\
$\mathrm{H}_{2} \mathrm{O}(\mathrm{ml})$ & & - \\
\hline
\end{tabular}

Die Menge Standardsubstanz soll der Konzentration an endogener 3-OH-A entsprechen, z. B. 2-3 $\mu$ g Standard bei einer Konzentration von ungefähr $1 \mu \mathrm{g} 3-\mathrm{OH}-\mathrm{A} / \mathrm{m} l$ Harn.

Unmittelbar nach Zugabe der Enzymlösung wird die Extinktion gegen einen Leerwert aus $1,0 \mathrm{~m} / \mathrm{Harn}+1 \mathrm{~m} /$ Puffer bei $360 \mathrm{~nm}$ abgelesen. Die Extinktion wird jede Minute abgelesen, so lange eine Zunahme zu beobachten ist, d. h. 10-20 Min. Während der Inkubation muß der Küvetteninhalt mehrmals gut gemischt werden.

Berecbnung

$$
\frac{\mathrm{A}}{(\mathrm{S}+\mathrm{A})-\mathrm{A}} \times 3=\mu \mathrm{g} / \mathrm{m} l 3-\mathrm{OH}-\mathrm{A}
$$

Bei einer Variation der Standardkonzentration muß der Faktor 3 entsprechend geändert werden.

b) Zur Bestimmung des 3-OH-K

Die Ansätze werden in $25 \mathrm{~m} /$ Erlenmeyerkolben mit Schliffstopfen durchgeführt und $3 \mathrm{Stdn}$. bei $38^{\circ}$ in einem Schüttel-Wasserbad in der in Tabelle 2 angeführten Zusammensetzung inkubiert.

Tab. 2

Ansätze zur Bestimmung des 3-OH-K im Harn Zugabe der Kyṇureninase in Substanz

\begin{tabular}{lccc}
\hline & $\begin{array}{c}\text { Analyse } \\
\text { (A) }\end{array}$ & $\begin{array}{c}\text { Analyse } \\
+ \text { Standard } \\
(\mathrm{S}+\mathrm{A})\end{array}$ & $\begin{array}{c}\text { "endogene" } \\
\text { 3-OH-A } \\
(\mathrm{E})\end{array}$ \\
\hline $\begin{array}{l}\text { Phosphatpuffer (ml) } \\
\text { Harn (ml) }\end{array}$ & 2,00 & & 2,00 \\
$\begin{array}{l}\text { Standardlösung (3-OH-K) } \\
\text { (ml) }\end{array}$ & 2,00 & $2, \overline{00}$ & 2,00 \\
$\begin{array}{l}\text { PLP-Lösung (ml) } \\
\text { Enzym (Kynureninase) }\end{array}$ & $0, \overline{10}$ & 2,00 & $0, \overline{10}$ \\
\multicolumn{1}{c}{ (mg) } & 15 & 15 & - \\
\hline
\end{tabular}

Anschließend an die Inkubation werden die Kölbchen im Wasserbad auf $20^{\circ}$ abgekühlt und der Inhalt filtriert, dann wird in jedem der Ansätze der Tabelle 2 die bereits vorhandene („endogene“) und die aus 3-OH-K gebildete 3-OH-A bestimmt. Dazu werden je $2 \mathrm{~m} l$ der Ansätze aus Tabelle $2 \mathrm{mit} 0,02 \mathrm{ml}$ der Cystein-FeLösung und $0,2 \mathrm{ml}$ der 3-Hydroxyanthranilsäureoxydaselösung versetzt und die Zunahme der Extinktion bei $360 \mathrm{~nm}$, wie unter a) beschrieben, verfolgt.

Berecbnung

$$
\frac{\mathrm{A}-\mathrm{E}}{(\mathrm{S}+\mathrm{A})-\mathrm{A}} \times 3=3-\mathrm{OH}-\mathrm{K} / \mathrm{m} / \text { Harn }
$$

Die Umrechnung auf die 24Stdn.-Harnmenge erfolgt unter Berücksichtigung der Verdünnung. Wird eine andere Standardkonzentration gewählt, muß der Faktor 3 in der Berechnungsformel entsprechend geändert werden.

\section{Zuverlässigkeit}

a) Zur Bestimmung der 3-OH-A

Bei 10 Bestimmungen der gleichen Harnproben fanden wir $0,45 \pm 0,025 \mu \mathrm{g} 3-\mathrm{OH}-\mathrm{A} / \mathrm{m} l$ Harn mit einem Variationskoeffi- zienten von 5,68. Zusatzversuche mit $3 \mu \mathrm{g}$ Standardsubstanz führten zu einer Ausbeute von $100 \pm 2,6 \%$. Weitere Angaben über die Spezifität und die Erarbeitung optimaler Reaktionsbedingungen sind der Originalarbeit zu entnehmen (4).

b) Zur Bestimmung des 3-OH-K

Der Zusatz von $1-4 \mu \mathrm{g} 3-\mathrm{OH}-\mathrm{K} / \mathrm{m} l$ zum Harn ergibt für diesen Bereich eine direkte Proportionalität zu den abgelesenen Extinktionen. Eine vollständige Umsetzung des Substrates ist damit gewährleistet.

\section{Ricbtigkeit}

Bei 10 Bestimmungen in der gleichen Harnprobe fanden wir eine Konzentration von $1,32 \pm 0,019 \mu \mathrm{g} 3-\mathrm{OH}-\mathrm{K} / \mathrm{ml}$ Harn (Mittelwert und Standardabweichung), Variationskoeffizient 4,46.

Zusatzversuche mit $1 \mu \mathrm{g}$ 3-OH-K ergaben eine Ausbeute von $1,0 \pm 0,022 \mu \mathrm{g}$ (Mittelwert und Standardabweichung), Variationskoeffizient $6,61(100 \pm 2,2 \%)$.

Zusatzversuche mit $3 \mu \mathrm{g}$ 3-OH-K ergaben eine Ausbeute von $3 \pm 0,016 \mu \mathrm{g}$ (Mittelwert und Standardabwieichung), Variationskoeffizient 1,62 (100 $\pm 0,53 \%)$.

\section{Sperifität}

Kynureninase setzt als Substrat 3-OH-K und Kynurenin um, durch die nachgeschaltete Reaktion wird jedoch immer nur die gebildete 3-OH-A erfaßt, so daß absolute Spezifität gegeben ist. Weitere Angaben zur Spezifität der Reaktion 3-OH-K $\longrightarrow$ 2-Akrolein3-aminofumarat s. SCHIEvelbeIN und BuchFINK (4).

\section{Ergebnisse}

Wir bestimmten bei 10 gesunden Personen die Ausscheidung von 3-OH-K und 3-OH-A. Die Ergebnisse sind aus Tabelle $3 \mathrm{zu}$ entnehmen.

Tab. 3

Die Ausscheidung von 3-OH-K und 3-OH-A bei Gesunden in $\mu \mathrm{g} / 24$ Stdn.-Harn

\begin{tabular}{ccccc}
\hline $\begin{array}{l}\text { Versuchs- } \\
\text { personen }\end{array}$ & $\begin{array}{c}\text { 3-OH-K } \\
\text { (frei) }\end{array}$ & $\begin{array}{c}\text { 3-OH-K } \\
\text { (gesamt) }\end{array}$ & $\begin{array}{c}\text { 3-OH-A } \\
\text { (frei) }\end{array}$ & $\begin{array}{c}\text { 3-OH-A } \\
\text { (gesamt) }\end{array}$ \\
\hline 1 & 478 & 712 & 248 & 677 \\
2 & 78 & 571 & 345 & 753 \\
3 & 110 & 256 & 284 & 391 \\
4 & 58 & 582 & 335 & 414 \\
5 & 461 & 447 & 258 & 346 \\
6 & 282 & 481 & 137 & 306 \\
7 & 348 & 349 & 280 & 378 \\
8 & 268 & 424 & 313 & 379 \\
$9 *$ & 463 & 4 & 325 & 570 \\
10 & 602 & 764 & 333 & 543 \\
\hline
\end{tabular}

* Die Gesamtausscheidung an 3-OH-K konnte aus äußeren Gründen bei dieser Versuchsperson nicht durchgeführt werden.

\section{Diskussion}

Vor kurzem beschrieben BeNAssI und Mitarbeiter (12) eine Methode zur Bestimmung von Tryptophanmetaboliten mit Hilfe von Absorption an Ionenaustauschersäulen, fraktionierte Elution und anschließende zweidimensionale Papierchromatographie, erneute Elution und anschließende kolorimetrische bzw. fluorometrische Bestimmung. Mit dieser Methode fanden die Autoren die folgenden Werte in $\mathrm{mg} / 24 \mathrm{Stdn}$ : $3-\mathrm{OH}-\mathrm{K} 0,5$ $(0,0-2,3)$ und für 3-OH-A 0,4 (0,01-1,1) Mittelwerte und Schwankungsbreite für den 24-Stdn.-Harn 20 gesunder Personen. Diese Werte stehen in sehr guter Ubbereinstimmung mit den von uns gefundenen Ausscheidungsgrößen und sind niedriger als die Werte in anderen älteren Arbeiten. Wir möchten dies als Folge der Verbesserung der Spezifität unserer und der Methode von BENASSI erklären. 
3-OH-K und 3-OH-A sind bei neutralem und bei alkalischem $\mathrm{pH}$ sehr instabil, so $\mathrm{da} ß$ bei einem $z$. B. durch Sulfonamidmedikation alkalisch reagierenden Harn eine Abnahme der Konzentration bereits in der Blase vorkommen kann. Sehr niedrige Harnwerte sind dadurch erklärbar (11).

Aus diesem Grunde ist auch die Bestimmung der sogenannten „endogenen 3-OH-A“ (Tab. 2) notwendig, denn diese kann in ihrer Konzentration wäh- rend der Inkubation mit Kynureninase ( 3 Stdn.) abgenommen haben. Eine kürzere Inkubationszeit für die Kynureninasereaktion ist unter unseren Bedingungen nicht möglich, da sonst ein vollständiger Umsatz des 3-OH-K nicht gewährleistet ist. Durch Verwendung aktiverer Enzympräparate könnte jedoch eine kürzere Inkubationszeit erreicht werden.

Wir danken der Wissenschaftlichen Forschungsstelle im Verband der Cigaretten-Industrie für die Unterstützung dieser Arbeit.

\title{
Literatur
}

1. Musajo, L., A. Spada und D. Coppint, J. biol. Chemistry 196, 185 (1952). - 2. Musajo, L. und C. A. Benassi, Adv. clin. Chem. 7, 63 (1964). - 3. Boyland, E., in: D. M. Wallace (ed.), Tumouts of the bladder. S. 83. E. \& S. Livingstone Ltd, Edinburg-London (1959). - 4. Schievelbein, H. und E. Buchfink, Clin. chim. Acta (Amsterdam) 18, 291 (1967). - 5. Bonner, D. M. und $\mathrm{C}_{\mathrm{H}}$. YANOFSKY, Proc. nat. Acad. Sci. USA 35, 576 (1949). - 6. Kuss, E., Hoppe-Seyler's Z. physiol. Chem. 345, 195 (1966). - 7. WeBER, F. und O. WIss, in: Hoppe-Seyler-Thierfelder (Hrsg).
Physiologisch- und pathologisch-chemische Analyse, 10. Aufl. Bd. VIjB, S. 806. Springer-Verlag, Berlin-Heidelberg-New York (1966). - 8. Wiss, O., Z. Naturforsch. 11b, 54 (1956). - 9. Weber, F. und O. Wiss, Hoppe-Seyler's Z. physiol. Chem. 304, 232 (1956). - 10. Wiss, O., H. Simmer und H. Peters, HoppeSeyler's Z. physiol. Chem. 304, 221 (1956). - 11. Pipkin, G. und J. U. Schlegel, Proc. Soc. exp. Biol. Med. 120, 592 (1965). 12. Benassi, C. A., F. M. Veronese und A. de Antoni: Clin. chim. Acta (Amsterdam) 17, 383 (1967).

\section{Die Verwendung von o-Nitrophenylbutyrat als Fehlerquelle bei der Messung der Reaktivierbarkeit alkylphosphatvergifteter Serumcholinesterase durch 2-PAM ${ }^{1}$ )}

\author{
Von M. Geldmacher - v. Mallinckrodt und I. Kaiser \\ Aus dem Institut für gericbtliche Medizin und Kriminalistik der Universität Erlangen-Nürnberg \\ (Direktor: Prof. Dr. Dr. E. Weinig)
}

(Eingegangen am 2. Februar 1968)

Das Vorgehen von MAIN, Miles und BraId zur Bestimmung der Se'rum-Cholinesteraseaktivität unter Verwendung von o-Nitrophenylbutyrat als Substrat ist nicht geeignet zur Messung der Reaktivierbarkeit alkylphosphatvergifteter Serumcholinesterase durch 2-PAM, da schon 2-PAM allein unter den Bedingungen der Reaktion das Substrat nichtkatalytisch unter Freisetzung von o-Nitrophenol spaltet.

The method by MAIN, MILES and BraId for determination of human serum cholinesterase activity with o-nitrophenylbutyrate as substrate is not suitable for measuring activity after reactivation of alcylphosphate-poisoned serum cholinesterase by 2-PAM, since 2-PAM alone - under the conditions of the reaction - splits the substrate non-catalytically and liberates o-nitrophenol.

Die Aktivität der Serumcholinesterase kann zur Diagnose bestimmter Krankheitsbilder und von Vergiftungen mit Cholinesteraseblockern wie z. B. E 605 herangezogen werden. Methodisch stehen eine große Anzahl verschiedener Verfahren zur Verfügung, die nicht alle Acetylcholin als Substrat verwenden (siehe z. B. Stumpf (1), Koelle (2).

MaIN, Mirles und Brarp (3) haben 1961 das o-Nitrophenylbutyrat als Substrat eingeführt. МсСомв, LAmotta und Wetstone (4) empfehlen dieses Substrat zur Erkennung atypischer Serumcholinesterasen. SzAsz (5) hat einen kritischen Vergleich zwischen Acetylthiocholin, Butyrylthiocholin und o-Nitrophenylbutyrat als Substrate zur Cholinesterase-Aktivitätsbestimmung im Serum durchgeführt.

\footnotetext{
1) Abletrrungen: 2-PAM = Pyridin-2-aldoxim-N-Methyljodid E $605=$ Diäthyl-p-Nitrophenylthiophosphat, DFP = Diisopropylfuorphosphat, TEPP $=$ Tetraäthylpyrophosphat.
}

Auf Grund des Vorkommens atypischer Serumcholinesterasen mit meist geringerer Aktivität (6), der starken intraindividuellen Unterschiede in der Aktivität der normalen Enzyme, des Einflusses von Krankheiten sowie der Möglichkeit einer Cholinesteraseblockierung nicht nur durch Alkylphosphate, sondern auch durch Carbamate und viele andere Medikamente haben Friedberg und SakaI (7) ein Verfahren zur in vitroReaktivierung alkylphosphatvergifteter Cholinesterasen mit 2-PAM ausgearbeitet. Sie empfehlen, mit einer beliebigen . Methode die Aktivität ohne und mit 2-PAMZusatz zu prüfen. Ergebe sich durch 2-PAM eine Aktivitätssteigerung, so liege mit Sicherheit eine Vergiftung mit einem organischen Phosphorsäureester vor.

FRIEDbERg und SAKaI (7) selbst verwendeten die manometrische Methode von AMMON unter Benutzung der Warburg-Apparatur, als Substrat diente Acetyl- 